\title{
Swing door technique for uncinectomy versus standard technique: a comparative study
}

\author{
Samar Pal Singh $\underline{\text { Yadav }}^{1}$, MBBS, Ms, Kuljeet $\underline{\text { Singh }}{ }^{1}$, MBBS, MS, Joginder Singh Gulia ${ }^{1}$, MBBS, MS, Anita $\underline{\text { Hooda }}{ }^{2}$, BDS, MDS
}

INTRODUCTION Uncinectomy can be performed using various methods. The aim of the present study was to compare the results and complications of uncinectomy and middle meatus antrostomy using the standard and swing door techniques during functional endoscopic sinus surgery.

METHODS In a prospective controlled study, 60 patients (aged 18-50 years) suffering from chronic maxillary sinusitis underwent functional endoscopic sinus surgery from January 2007 to December 2008 at a tertiary care centre. The patients were randomly divided into two groups of 30: Group A underwent uncinectomy using the standard technique, while Group B underwent uncinectomy using the swing door technique.

RESULTS Group B showed more improvement in symptoms, with a mean visual analogue scale score of $80.58 \pm 14.34$ compared to $78.50 \pm 16.63$ in Group A. Both groups had no major complications. At the end of Week 2 , minor complications were observed in $8(26.7 \%)$ of the patients from Group A and 2 (6.7\%) from Group B. By the sixth week, the minor complication rate was 1 (3.3\%) and 0 ( $0 \%)$ in Group A and Group B, respectively. When compared statistically during the second week using chi-square test, the difference in the minor complication rate was found to be statistically significant $\left(p<0.05, \chi^{2}=4.81\right)$, with lower incidence of complications in Group B.

CONCLUSION The swing door technique for uncinectomy produces good postoperative results, with fewer complications, as compared to the standard technique.

Keywords: chronic maxillary sinusitis, functional endoscopic sinus surgery, middle meatus antrostomy, swing door uncinectomy, uncinectomy

\section{INTRODUCTION}

Functional endoscopic sinus surgery (FESS) is now the gold standard for the management of refractive sinonasal diseases. The standard technique for performing uncinectomy and middle meatus antrostomy (MMA) is effective but associated with risks such as lamina papyracea lesions, orbital haematoma, loss of vision, nasolacrimal duct stenosis and obstruction of maxillary sinus ostium. ${ }^{(1,2)}$ Wormald and McDonogh proposed a new method for performing uncinectomy, termed the swing door technique, that allows the uncinate to be removed flush with the lateral nasal wall and easy identification of the natural ostium of the maxillary sinus while avoiding complications. ${ }^{(3)}$

We present the results of a prospective controlled study that was undertaken to determine the efficacy of the swing door technique, as compared to the standard technique of uncinectomy, for uncinectomy and MMA.

\section{METHODS}

The study was conducted at the Department of Otolaryngology, Pandit Bhagwat Dayal Sharma University of Health Sciences, Rohtak, India, between January 2007 and December 2008. A total of 60 patients of either gender and aged $18-50$ years with chronic maxillary sinusitis were included. Patients were randomly divided into two groups of 30 using a periodic random number. For Group A patients, uncinectomy was performed using the standard technique, and for Group B patients, uncinectomy was performed using the swing door technique.

Patients suffering from sinusitis for more than six months and diagnosed with chronic maxillary sinusitis were selected. Computed tomography $(\mathrm{CT})$ of the paranasal sinus was done, and only patients with disease limited to the maxillary sinus were recruited, so that the total number of eligible patients was limited. Patients with involvement of other sinuses or anatomic variations of the osteomeatal area were excluded from the study. Patients with symptomatic deviated nasal septum were also excluded, and only patients with mild deviated nasal septum that was deemed not likely to be a cause of any problem during FESS were included in the study. Routine blood and urine tests, pre-anaesthetic check-ups and xylocaine sensitivity testing was done for all patients. Written informed consent was obtained from all participants. All patients were operated on by the senior surgeon under local anaesthesia.

Patients were placed in the supine position with the head in a slightly elevated position. Nasal packs were removed. The anterior part of the inferior turbinate, anterior part of the middle turbinate, uncinate process and posterior part of the middle meatus were injected with $2 \%$ xylocaine with 1:100,000 epinephrine under endoscopic visualisation.

For Group A patients, uncinectomy and MMA was performed using the standard technique, in which the base of the uncinate was incised from the superior attachments to inferior attachments

${ }^{1}$ Department of Otolaryngology, ${ }^{2}$ Department of Oral Anatomy, Pandit Bhagwat Dayal Sharma University of Health Sciences, Rohtak, Haryana, India Correspondence: Dr Joginder Singh Gulia, Professor, Department of Otolaryngology, Pandit Bhagwat Dayal Sharma University of Health Sciences, House No. 20/9J, Medical Campus, 124001 Rohtak, Haryana, India. jsgulia@hotmail.com 
using a sickle knife. The incision was given in the groove where the uncinate joins the lateral nasal wall. During the incision, the uncinate was pushed medially using the sickle knife to facilitate its removal with a pair of Blakesley forceps. The incision was continued inferiorly and taken further posteriorly along the uncinate's insertion into the base of the inferior turbinate. The uncinate was grasped superiorly with the Blakesley forceps and rotated down during removal to reveal the natural ostium of the maxillary sinus and to open up the middle meatus.

For Group B patients, uncinectomy was performed using the swing door technique. In this technique, the uncinate was identified by palpation. A sickle knife was used to incise horizontally across the uncinate in the axilla of the middle turbinate. A pair of backbiting forceps was passed beyond the uncinate to engage it. In patients with a normal-sized uncinate, a further bite by the forceps was done to cut the uncinate on its insertion on the lateral nasal wall. The punch was rotated superiorly almost vertically to bring it medial to the nasolacrimal duct. The uncinate was engaged as low as possible to remove most of its horizontal portion to expose the natural ostium. A ball probe was passed posterior to the edge of the cut uncinate and was pulled anteriorly to fracture the uncinate at its insertion to the lateral nasal wall. This swinging uncinate was grasped using an angled pair of $45^{\circ}$ Weil-Blakesley forceps and was removed in toto. The residual part of the uncinate, if any, was removed using back-biting forceps. MMA was done as in the standard technique. During surgery, the patient was evaluated for pain in the eye, injury to the middle turbinate and fat prolapse.

At the end of surgery, Soframycin ointment-coated light anterior nasal pack was put on the middle meatus. Patients were discharged on the second day of surgery after pack removal. Patients were given oral antibiotics, analgesics, anti-histaminics, and saline and $0.1 \%$ xylometazoline nasal drops thrice a day for one week. Patients were then given fluticasone $0.05 \%$ nasal spray, alkaline nasal douching and saline nasal drops for two weeks.

Follow-up assessment was performed at the end of the second week. Patients were subjectively reviewed for symptomatic relief using visual analogue scale (VAS) scores, and postoperative symptoms were compared to preoperative ones. The final assessment was done at the end of the sixth week on the basis of: (a) subjective criteria, where complaints of watering from the eye (i.e. epiphora) and any other nasal complaint were noted; and (b) objective criteria (on nasal endoscopy), where synechiae formation, patency of antrostomy and remnant of the uncinate process were noted. The VAS is a 100-mm self-rating scale in which scores range from 0 to 100 , where 0 indicates worst symptoms and 100 indicates complete freedom from symptoms. In this scale, there are an infinite number of points between extremes, so that the symptomatic assessment can be correlated on a subjective basis.

At the end of the study, data was collected and analysed using Student's $t$-test and chi-square test.

\section{RESULTS}

The 60 patients were aged between 18 and 50 years. The mean age of the patients in Group A was 29.13 years and that of Group B
Table I. Duration of symptoms among patients undergoing uncinectomy.

\begin{tabular}{|llll|}
\hline Duration & \multicolumn{3}{c|}{ No. (\%) } \\
\cline { 2 - 4 } & Total $(\mathbf{n = 6 0 )}$ & Group A $(\mathbf{n = 3 0 )}$ & Group B $(\mathbf{n = 3 0 )}$ \\
\hline $6 \mathrm{mth}-1 \mathrm{yr}$ & $15(25.0)$ & $5(16.7)$ & $10(33.3)$ \\
\hline $1-2 \mathrm{yr}$ & $8(13.3)$ & $5(16.7)$ & $3(10.0)$ \\
\hline $2-3 \mathrm{yr}$ & $9(15.0)$ & $7(23.3)$ & $2(6.7)$ \\
\hline$>3 \mathrm{yr}$ & $28(46.7)$ & $13(43.3)$ & $15(50.0)$ \\
\hline
\end{tabular}

Group A: standard technique; Group B: swing door technique

was 26.03 years. Of 30 patients in Group A, 25 were men and five were women (male-to-female ratio 5:1). Of 30 patients in group $\mathrm{B}, 18$ were men and 12 were women (male-to-female ratio $3: 2)$. Overall, nearly half $(\mathrm{n}=28,46.7 \%)$ of the patients had preoperative symptoms for over three years (Table I). Among the remaining patients, $15(25.0 \%)$ had symptoms for six months to one year, 8 (13.3\%) had symptoms for $1-2$ years and 9 (15.0\%) had symptoms for 2-3 years. Nasal obstruction and postnasal drip were observed in all patients; rhinorrhoea or nasal discharge was also a common symptom.

Among the 60 patients who underwent an uncinectomy, anterior rhinoscopy revealed deviated nasal septum in 54 (90.0\%) patients. Other significant findings were discharge in the middle meatus ( $\mathrm{n}=33,55.0 \%$ ) and hypertrophy of the inferior turbinate $(\mathrm{n}=18,30.0 \%)$. Posterior nasal discharge was seen in $36(60.0 \%)$ patients. Hypertrophy of the posterior end of the inferior turbinate ( $\mathrm{n}=18,30.0 \%$ ) was another key finding. The presence of discharge and congestion of the posterior pharyngeal wall were major clinical findings on oral examination in 30 (50.0\%) and $24(40.0 \%)$ patients, respectively. Examination of the ears was within normal limits for all except $3(5.0 \%)$ patients, who had ear discharge with safe central perforation.

Coronal and axial view CT of the paranasal sinuses was done for all patients. Obstruction of the osteomeatal complex was the most common finding, seen in 58 (96.7\%) patients. Other findings were mucosal hypertrophy of the maxillary sinus, which was seen in $42(70.0 \%)$ patients, and polyp in the maxillary sinus, found in $19(31.7 \%)$ patients.

No major complications were observed in either of the two patient groups. At the end of the second week, there were $8(26.7 \%)$ minor complications in Group A (remnant of uncinate process, $\mathrm{n}=2$; blocked MMA, $\mathrm{n}=3$; adhesions, $\mathrm{n}=3$ ) and $2(6.7 \%)$ minor complications in Group B (adhesions, $\mathrm{n}=2$ ). At the end of the sixth week, the minor complication rate was $1(3.3 \%)$ for Group A and $0(0 \%)$ for Group B. Statistical analysis using chi-square test after the second week showed that the difference in the minor complication rates of Groups $A$ and B was statistically significant $\left(p<0.05, \chi^{2}=4.81\right.$ ), with a lower incidence of complications in Group B.

Postoperative follow-up assessment at six weeks showed that among 30 patients in Group A with nasal obstruction, $18(60.0 \%)$ patients had $100 \%$ relief, $8(26.7 \%)$ patients had $90 \%$ relief and $3(10.0 \%)$ patients had $80 \%$ relief, according to their VAS scores (Table II). Out of 30 patients, 12 (40.0\%) with 
Table II. Postoperative assessment of symptoms at Week 6 using VAS.

\begin{tabular}{|c|c|c|c|c|}
\hline \multirow[t]{2}{*}{ Symptom } & \multicolumn{2}{|c|}{ Group A $(n=30)$} & \multicolumn{2}{|c|}{ Group B $(n=30)$} \\
\hline & VAS score & No. (\%) & VAS score & No. (\%) \\
\hline \multirow{5}{*}{$\begin{array}{l}\text { Nasal } \\
\text { obstruction }\end{array}$} & 100 & $18(60.0)$ & 100 & $24(80.0)$ \\
\hline & 90 & $8(26.7)$ & 90 & $1(3.3)$ \\
\hline & 80 & $3(10.0)$ & 80 & $1(3.3)$ \\
\hline & 50 & $1(3.3)$ & 70 & $1(3.3)$ \\
\hline & & & 50 & $3(10.0)$ \\
\hline \multirow{6}{*}{$\begin{array}{l}\text { Postnasal } \\
\text { drip }\end{array}$} & 100 & $1(3.3)$ & 100 & $1(3.3)$ \\
\hline & 90 & $3(10.0)$ & 90 & $9(30.0)$ \\
\hline & 80 & $6(20.0)$ & 80 & $12(40.0)$ \\
\hline & 70 & $12(40.0)$ & 70 & $5(16.7)$ \\
\hline & 60 & $6(20.0)$ & 60 & $1(3.3)$ \\
\hline & 50 & $2(6.7)$ & 50 & $2(6.7)$ \\
\hline \multirow{5}{*}{$\begin{array}{l}\text { Nasal } \\
\text { discharge* }\end{array}$} & 100 & $1(4.2)$ & 100 & $1(3.3)$ \\
\hline & 90 & $6(25.0)$ & 90 & $10(33.3)$ \\
\hline & 80 & $9(37.5)$ & 80 & $12(40.0)$ \\
\hline & 70 & $3(12.5)$ & 70 & $6(20.0)$ \\
\hline & 60 & $5(20.8)$ & 60 & $1(3.3)$ \\
\hline
\end{tabular}

*24 patients in Group A. Group A: standard technique; Group B: swing door technique; VAS: visual analogue scale

postnasal drip reported $70 \%$ improvement and 9 (37.5\%) of 24 patients with nasal discharge had $80 \%$ improvement. For patients in Group B with nasal obstruction, at the six-week follow-up, $24(80.0 \%)$ patients had $100 \%$ relief, 1 (3.3\%) patient had $90 \%$ relief, 1 (3.3\%) patient had $80 \%$ relief and 1 (3.3\%) patient had $70 \%$ relief according to VAS scores. Out of 30 patients, $12(40.0 \%)$ with postnasal drip had $80 \%$ improvement and 12 (40.0\%) of 30 patients with nasal discharge had $80 \%$ improvement.

In Group A, complete eradication of symptoms (i.e. a VAS score of 100$)$ was achieved by $18(60.0 \%)$ of 30 patients with nasal obstruction, 1 (3.3\%) of 30 patients with postnasal drip and 1 (4.2\%) of 24 patients with nasal discharge. In Group B, a VAS score of 100 was achieved for $24(80.0 \%)$ of 30 patients with nasal obstruction, 1 (3.3\%) of 30 patients with postnasal drip and $1(3.3 \%)$ of 30 patients with nasal discharge.

Postoperative improvement in symptoms after six weeks was found to be highly significant statistically $(p<0.001)$ in both groups. The mean VAS scores for Group A and Group B were $78.50 \pm 16.63$ and $80.58 \pm 14.34$, respectively, suggesting that Group B patients had better symptomatic improvement. However, when the postoperative improvement in symptoms after six weeks of Group A and Group B patients was compared using Student's $t$-test, the difference was not statistically significant ( $p>0.05)$.

\section{DISCUSSION}

The physiologic foundation for FESS is that the major sinuses are capable of reversal of mucosal disease if ventilation is reestablished. Endoscopic sinus surgery provides easy access and better visualisation of the structures of the lateral nasal wall, thereby ensuring better surgical results. This technique does not alter the normal physiological functioning of sinus mucosa, and is thus known as FESS. ${ }^{(1,2)}$ Although the standard method for performing uncinectomy and MMA is effective, it may be associated with risks such as lamina papyracea lesion, orbital haematoma, loss of vision, nasolacrimal duct stenosis and obstruction of the maxillary sinus ostium. ${ }^{(3)}$ The extent of sinonasal inflammatory disease, important anatomic landmarks and their variations can be easily detected on $\mathrm{CT}$, which provides a reliable roadmap for endoscopic surgery. ${ }^{(4,5)}$

The uncinate is usually recognised by palpation and movement of its free edge. In the standard technique, the incision in the uncinate is made flush with the lateral nasal wall, and therefore there is a risk of penetrating the orbit, especially if anatomical variations of the uncinate are present. ${ }^{(6,7)}$ In the swing door technique for uncinectomy, as proposed by Wormald and McDonogh, the uncinate can be removed flush with the lateral nasal wall and there is easy identification of the natural ostium of the maxillary sinus. ${ }^{(3)}$

In our study, we compared outcomes of patients who underwent uncinectomy and MMA using the swing door technique (Group B) with those for whom the standard technique of uncinectomy (Group A) was used. The mean age of patients in Group B was slightly less than that in Group A. Overall, there was a predominance of men, with 43 men and 17 women (male-to-female ratio 2.5:1) in the study. This was similar to the observations made by Bolger et $\mathrm{al}^{(5)}$ and Sinha ${ }^{(8)}$ in their studies.

Common symptoms in our study were nasal obstruction and postnasal drip (100\% in both groups), and nasal discharge (90\% in both groups). Symptomatology in our patients was comparable to that in a study by Kennedy ${ }^{(6)}$ On the other hand, Salam and Cable observed that postnasal discharge $(88.2 \%)$, nasal obstruction $(84.3 \%)$, headache $(78.4 \%)$ and hyposmia $(11.76 \%)$ were major symptoms in a study of 51 patients with chronic maxillary sinusitis who underwent MMA.(7) Bhattacharyya, who studied the differences in symptoms and disease severity among 155 patients with nasal septal deviation and chronic rhinosinusitis, concluded that these patients had higher severity scores for nasal symptoms, and that nasal obstruction and nasal discharge were the most severe symptoms. ${ }^{(9)}$

Besides the clinical and radiological findings, the duration of symptoms is very important for diagnosis. Lund and Kennedy used persistent symptoms and signs for more than eight weeks as the criteria for chronic sinusitis. ${ }^{(10)}$ However, Litton has suggested a time interval of three months, ${ }^{(11)}$ while Benninger et al was in favour of inflammation of the mucosa of the nose and paranasal sinuses for at least 12 consecutive weeks. ${ }^{(12)}$ In the present study, most $(46.7 \%)$ patients had symptoms for over three years and none of our patients had symptoms for less than six months.

Common clinical signs among our patients were deviated nasal septum $(90.0 \%)$, posterior nasal discharge $(60.0 \%)$, nasal discharge (55.0\%), congested posterior pharyngeal wall (42.5\%) and hypertrophy of the inferior turbinate (30.0\%). Sinha reported the presence of mucus or muco-pus in the middle meatus in $76 \%$ of 42 patients in his series. ${ }^{(8)}$

$\mathrm{CT}$, with its excellent capability for displaying bone and soft tissues, is the current diagnostic modality of choice for evaluating the osteomeatal complex and provides precise guidance for 
therapeutic endoscopic instrumentation. ${ }^{(4,5,13)}$ In the present study, the most common findings were osteomeatal complex obstruction $(96.7 \%)$, deviated nasal septum $(90.0 \%)$, mucosal hypertrophy (70.0\%) and maxillary polyps (31.7\%).

In our study, 2 (6.7\%) patients from Group A developed periorbital swelling. Levine, who evaluated 250 patients undergoing FESS, concluded that $8.3 \%$ of patients developed minor complications and $0.7 \%$ developed major complications. ${ }^{(14)}$ Vleming et al reported orbital complications of endoscopic sinus surgery, including lamina papyracea lesions, and intraorbital and retrobulbar haematomas. ${ }^{(15)}$ In our study, $1(3.3 \%)$ patient had epiphora during the postoperative period. Serdahl et al reported nasolacrimal duct injury and obstruction after endoscopic sinus surgery. It is likely that vigorous anterior enlargement of the middle meatus might be the cause for nasolacrimal duct obstruction. ${ }^{(16)}$ In order to avoid and minimise trauma to the lacrimal apparatus, Bolger et al suggested a modification of the standard uncinectomy method. ${ }^{(17)}$ Unlu et al concluded that lacrimal drainage system injury might occur to various extents during FESS and advised a modified technique for uncinectomy similar to the swing door technique to minimise these complications. ${ }^{(18)}$

In Group B, there were postoperative adhesions at first followup in $2(6.7 \%)$ patients, which were relieved. Patients improved symptomatically when assessed using VAS, and postoperative symptomatic improvement at the sixth week was found to be highly significant $(p<0.001)$. Antrostomy was patent in all patients and no remnants of the uncinate process were seen in any patient. Thus, in our study, the swing door technique for uncinectomy was associated with lower postoperative complication rates.

Puranik and El-Sheikha conducted a postal survey and analysed results from 458 completed questionnaires they received from surgeons who performed FESS. ${ }^{(19)}$ They concluded that $18.9 \%$ of the surgeons surveyed had no preference for any uncinectomy technique and $21.17 \%$ performed either the Stammberger or swing door technique. However, most surgeons were of the opinion that for both techniques, training was needed to perform the procedure effectively without any problems and, at least initially, there was always a learning curve. ${ }^{(19)}$
To conclude, in the present study, it was observed that the swing door technique for uncinectomy gives good postoperative results, with fewer complications, when compared to the standard technique. Where India is concerned, FESS is in the developing phase, except at premier institutes. Young surgeons are not well trained in endoscopic surgery, and therefore the swing door technique for uncinectomy is better suited for use, yielding good results with minimum complications.

\section{REFERENCES}

1. Stammberger $\mathbf{H}$. Endoscopic endonasal surgery--concepts in treatment of recurring rhinosinusitis. Part I. Anatomic and pathophysiologic considerations. Otolaryngol Head Neck Surg 1986; 94:143-7.

2. Stammberger $\mathrm{H}$. Endoscopic endonasal surgery--concepts in treatment of recurring rhinosinusitis. Part II. Surgical technique. Otolaryngol Head Neck Surg 1986; 94:147-56.

3. Wormald PJ, McDonogh M. The 'swing-door' technique for uncinectomy in endoscopic sinus surgery. J Laryngol Otol 1998; 112:547-51.

4. Shroff MM, Shetty PG, Navani SB, Kirtane MV. Coronal screening sinus CT in inflammatory sino-nasal disease. Indian J Radiol Imag 1996; 6:3-17.

5. Bolger WE, Butzin CA, Parson DS. Paranasal sinus bony anatomic variations and mucosal abnormalities: CT analysis for endoscopic sinus surgery. Laryngoscope 1991; 101(1 Pt 1):56-64.

6. Kennedy DW. Prognostic factors, outcomes and staging in ethmoid sinus surgery. Laryngoscope 1992; 102(12 Pt 2 Suppl 57):1-18.

7. Salam MA, Cable HR. Middle meatal antrostomy: long-term patency and results of chronic maxillary sinusitis. A prospective study. Clin Otolaryngol Allied Sci 1993; 18:135-8.

8. Sinha DK. Nasal and sinus endoscopy in opaque maxillary antrum. Indian J Otolaryngol Head Neck Surg 1993; 45:145-6.

9. Bhattacharyya N. Symptom and disease severity differences between nasal septal deviation and chronic rhinosinusitis. Otolaryngol Head Neck Surg 2005; 133:1 173-7.

10. Lund VJ, Kennedy DW. Staging of rhinosinusitis. Otolaryngol Head Neck Surg 1997; 117(3 Pt 2):S35-40.

11. Litton WB. Acute and chronic sinusitis. Otolaryngol Clin North Am 1971; 4:25-37.

12. Benninger MS, Ferguson BJ, Hadley JA, et al. Adult chronic rhinosinusitis: definitions, diagnosis, epidemiology and pathophysiology. Otolaryngol Head Neck Surg 2003; 129(3 Suppl):S1-32.

13. Zinreich SJ, Kennedy DW, Rosenbaum AE, et al. Paranasal sinuses: CT imaging requirements for endoscopic surgery. Radiology 1987; 163:769-75.

14. Levine HL. Functional endoscopic sinus surgery: evaluation, surgery, and follow-up of 250 patients. Laryngoscope 1990; 100:79-84.

15. Vleming $M$, Middelweerd R, de Vries N. Complication of endoscopic sinus surgery. Arch Otolaryngol Head Neck Surg 1992; 118:617-23.

16. Serdahl CL, Berris CE, Chole RA. Nasolacrimal duct obstruction after endoscopic sinus surgery. Arch Ophthalmol 1990; 108:391-2.

17. Bolger WE, Parsons DS, Mair EA, Kuhn FA. Lacrimal drainage system injury in functional endoscopic sinus surgery. Incidence, analysis, and prevention. Arch Otolaryngol Head Neck Surg 1992; 118:1179-84.

18. Unlu HH, Goktan C, Aslan A, Tarhan S. Injury to the lacrimal apparatus after endoscopic sinus surgery: surgical implications from active transport dacryocystography. Otolaryngol Head Neck Surg 2001; 124:308-12.

19. Puranik V, El-Sheikha A. Uncinectomy: Stammberger or swing-door technique? Eur Arch Otorhinolaryngol 2007; 264:1151-5. 